\title{
Performance analysis of the ZigBee networks in 5G environment and the nearest access routing for improvement
}

\begin{abstract}
The ZigBee network is widely studied and deployed recently because its low cost and simplicity features. However, the power consumption issue needs a further improvement since the application requirements are not fully satisfied. The emerging $5 \mathrm{G}$ communication technology is characterized by the smarter devices and the native support for the M2M communication. On that basis, the 5G terminals are capable of joining the existing ZigBee networks and have the potential to improve the data transmission. In this paper, we investigate the performance of the ZigBee networks in the $5 \mathrm{G}$ environment for different scenarios. Then a nearest access routing (NAR) algorithm based on the physical depth is proposed for different communication types. To reduce the loads in ZigBee networks, the data flow in the neighborhood of 5G terminals is gathered and transmitted via the IP networks. The simulation results showed that NAR effectively share the communication in ZigBee networks. It leads to better performances with higher packet delivery ratio, less hop counts from ZigBee devices, lower latency, fewer packets sent by ZigBee nodes and zero routing overheads.
\end{abstract}

Keywords: ZigBee; 5G; routing; performance analysis; mobility

\section{Introduction}

ZigBee technology is characterized by low cost, low power, low data rate, and simplicity [1, 2]. Due to these attractive features, recently ZigBee network is widely studied and deployed. There are two options for the ZigBee routings, the ZigBee tree routing (ZTR) and ad-hoc on demand distance vector (AODV) [3]. ZTR is based on the hierarchy, and featured by its simplicity and reliability, although it is not always efficient [4]. The AODV routing is able to find the global shortest path by flooding the routing request, while it often results in more consumption and higher latency. The hybrid routing mechanism is designed for different requirements in the data transmission. However, limited by the insufficient resources in the ZigBee devices, the described routing protocols cannot fully address power consumption issues [5-9]. Besides optimizing the current network formation and protocols, it is another way that to improve the performance with the help of the deployed environment, which includes the coming $5 \mathrm{G}$ communication network.

5G Technology indicates the next most important stage of mobile communication standards beyond the 4G. Due to the extremely higher aggregate data rates and the much lower latencies required, along with other breakthrough technologies, the smarter devices and the native support for the M2M communication are two of the most important features in the 5G communication network [10-12]. The smarter devices will be able to have access to different wireless technologies at the same time [13]. Meanwhile, a native inclusion of M2M communication in 5G involves satisfying three fundamentally different requirements associated with different classes of low-data-rate services: support of a massive number of low-rate devices, sustaining a minimal data rate in virtually all circumstances, and very-low-latency data transfer [14]. Based on the above functionalities, it is reasonable to take the ubiquitous smarter terminals into account to improve 
the performance of ZigBee networks in the $5 \mathrm{G}$ environment.

A sample deployment is shown in Fig.1. Thanks to the inclusion of M2M communication function, the $5 \mathrm{G}$ terminals which locate within the coverage of the ZigBee networks are able to join the existing ZigBee networks. Compare with the ZigBee nodes, owing to the adequate energy, storage, processing and bandwidth resources in the mobile devices; they have the potential to improve the data transmission in the ZigBee network. Different from the existing relay or external node, note that the $5 \mathrm{G}$ terminals could also access the IP network. That means they are capable of communicating with the sink and management device directly without consuming the ZigBee bandwidth, and the ZigBee packets can be gathered and conveyed in the internet links. Our work is to develop a routing scheme for $5 \mathrm{G}$ devices to share the traffic in ZigBee network as much as possible so that the overhead in the ZigBee networks can be lightened effectively.

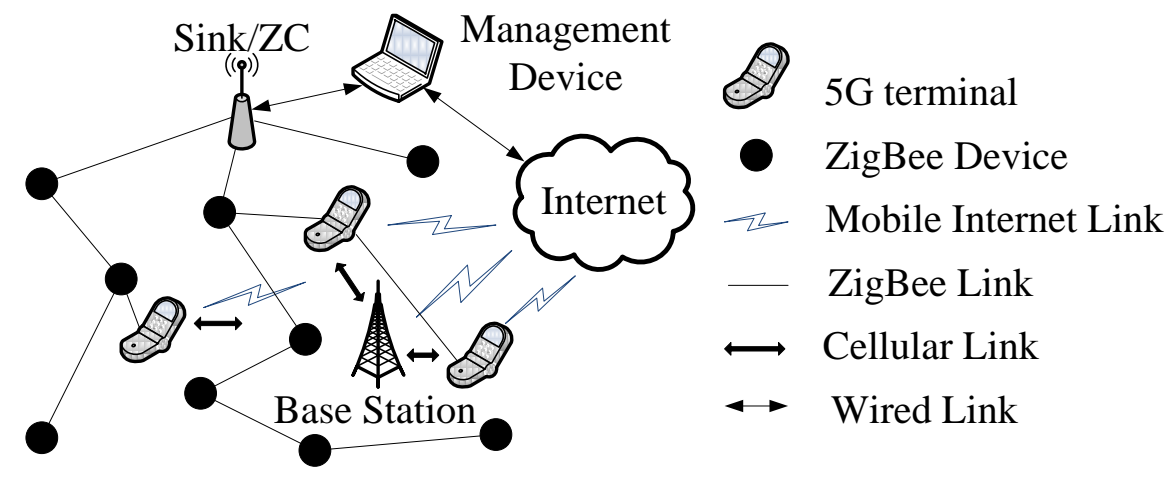

Fig. 1. The ZigBee network in $5 \mathrm{G}$ environment

To the best of our knowledge, this is the first work on the performance improvement for ZigBee networks in the $5 \mathrm{G}$ environment. Considering the dual ZigBee routing algorithms, although the 5G terminal could improve the routing discovery, the related links may not last long because its location keeps changing. Moreover, for the large scale networks or the nodes with mobility, ZTR has a much better performance $[15,16]$. Thus, in this paper, we exploit the ZTR-based method to find the paths via $5 \mathrm{G}$ devices. A nearest access routing algorithm based on the physical depth is proposed to optimize the data transmission in the heterogeneous network.

The remainder of this paper is organized as follows: Section 2 briefly summarizes the related work, and Section 3 introduces some preliminary knowledge in the ZigBee networks. Two scenarios of the heterogeneous networks are described and analysed in the Section 4. On that basis, the nearest access routing protocol is proposed in Section 5. Section 6 shows the simulation results and the evaluation of our method. The conclusion is given in Section 7.

\section{Related work}

Some studies on the improvement of the ZTR have been proposed due to its simplicity and low cost [17]. STR [18] is one of the most significant works in the literature. The network address is recommended to assign based on the Distributed Address Allocation Mechanism, by which the hierarchy of the ZigBee network is inherently formed. In this case, once the network address of a certain device is known, the topology depth can be determined. Furthermore, given the addresses of the source and the destination nodes, the hop counts via ZTR can be calculated. In the STR, based on the neighbour table maintenance which is specified by the ZigBee, each sending device 
may decide the next hop receiver which has the minimum remaining hop counts via ZTR among all the neighbour candidates. The work shows that the STR achieves the compatible performance to the Z-AODV which always gets the global shortest path with limited cost. On that basis, several improvements on the STR have been also investigated. An extended STR routing method (ESTR) to improve the packet delivery ratio (PDR) and the latency is presented in [19]. It is claimed that the ESTR increases the robustness and reduces the delay, but in our opinions, its effectiveness remains questionable. The algorithm to find the link is not explained or described but just given some codes without clear comments. Furthermore, based on the given figures, a regular distribution which is difficult to satisfy in real world is required. In [20], the authors design an opportunistic STR (OSTR) to enhance the reliability of the ZigBee network. Instead of determining a next hop node before sending a frame, a competing retransmission mechanism based on the priority of remaining hops is introduced to opportunistic route the packet. To increase the bandwidth utilization, [21] proposes an adoptive-parent-based frame work for the ZigBee cluster-tree topology. When a node is to send data in a high bit rate (even exceeds the upper bound specified in the ZigBee), besides its primal parent, the packet is distributed to the adopt parent nodes which locate in the neighbourhood to transmit in a multipath manner. The network throughput is thus improved and the algorithm is compatible with the existing ZigBee specification without introducing any extra communication. Nevertheless, the cross-link problem, the effectiveness in low density network and the interferences in high density networks which may limit its improvement are not well studied yet. A routing method which is similar as the STR is proposed in [22]. The authors firstly evaluate the different routing methods by OPNET simulator, and propose a modified ZTR routing (ZBR-M) algorithm. In the ZBR-M, the next hop node is not specified, but the packet is broadcast to all the neighbours. The receiver retransmits the packet only when it provides a shorter remaining path and no duplication sent by other device is overheard. Due to the carrier sense multiple access collision avoidance (CSMA/CA) mechanism, the link in the ZBR-M is random and may not be optimal for tree cluster topology. Regarding the node mobility issue, a ZigBee node deployment and tree construction framework is discussed in [23]. Based on the real application, the movement pattern of the node is categorized and considered during the network construction to make the router locate along the trajectory of the mobile device with the maximum edge numbers in a graph. By adopting an overhearing mechanism, the node movement is well estimated and the packet loss is effectively suppressed. However, owing to the specified network architecture, it does not get a universal solution for the general ZigBee networks.

The mentioned works mostly focus on the routing optimization for the ZTR, while our work takes the deployment environment into account. Besides the investigation of the use pattern for the DAAM network address and neighbour table information in 5G networks, the features of $5 \mathrm{G}$ devices and the routing improvement are also studied in this paper.

On the other hand, with the rapid development of different wireless technologies, it has been more common that multiple overlapping WSNs construct on the same area. Thus there have been lots of researches on the routing improvement for the wireless heterogeneous sensor networks (WHSN) emerging in the past few years [24-26]. In [27] proposes a routing protocol using the heterogeneous ZigBee nodes to minimize the routing overhead. By integrating devices with different transmission ranges, the router device extends its coverage to reduce the loads. Note that this work is only heterogeneous for hardware, but overall it is still a homogeneous ZigBee 
network. [28] proposes a tree-based approach for the internet connectivity of mobile ad hoc networks (MANET), which is organized in a hierarchical topology similar as the one in ZigBee. However, the nodes require much more resources than the ZigBee devices. An integrated gateway node control protocol for home area network (HAN) is discussed in [29]. By developing the steps including initial flooding, clustering, gateway selection, gateway integration and linking, the network lifetime is extended. Whereas, only the many-to-one link from sensors to the sink focuses on the algorithm. And it also requires a high node density to form the integrated gateway node. In [30], a fair cooperative routing method for overlapped heterogeneous networks is studied. A shared energy pool is introduced and used as a broker to fairly allocate the energy. The cooperative forwarding leads to maximum energy efficiency for all the composing networks. What is different in our work is that we only try to minimize the cost for one component, the ZigBee network. A hybrid grid routing protocol (HGRP) for heterogeneous hierarchical wireless networks is proposed in [31]. According to the location, the network deploying area is properly divided into grids, each of which covers at least one backbone routing nodes and some ordinary nodes. Compared with common ZigBee routing protocols, the HGRP achieves lower routing cost and energy consumption. However, the high density and the uniformly distribution of backbone nodes may be demanding for most applications. In our work, we do not have special requirements for the auxiliary devices distribution. In [32], a routing protocol based on topologies for HSN named as RouT is discussed. By classifying the devices into L-sensors and H-sensor, which represent the nodes with low and high hardware capacities respectively, the algorithm establishes different logical topologies upon the same physical topology on the basis of different link numbers among H-sensors. The simulation results show that the RouT leads to a higher diversity and reliability in the HSNs, but it does not fit for our work. Not only because the parallel links require a regular node deployment, but also frequent topology changing the mobile devices may cause.

\section{ZigBee networks}

\subsection{Overview of ZigBee}

Three device types are defined in the ZigBee specification: ZigBee coordinator (ZC), ZigBee routers (ZR), and ZigBee end devices (ZED). The ZC, which usually works as the sink node and is connected to the management device, is responsible for starting a new network. The $\mathrm{ZR}$, as well as the $\mathrm{ZC}$, is routing capable, while the ZED can't take part in routing and have to rely on their corresponding ZigBee parent nodes for data transmission. The deployed ZigBee devices self-organize the network, and then the topology changes can be automatically reflected in the network configuration [1].

ZigBee devices support the link quality indication (LQI) measuring whenever they receive a packet. The LQI measurement is a characterization of the strength and/or quality of the frame, the acquiring costs no more extra calculation and communication [1].

\subsection{Address allocation}

The Distributed Address Allocation Mechanism (DAAM) is recommended for the network address assignment to build the hierarchy. The parameter $\mathrm{Cm}$ means the number of children nodes for a routing capable device in maximum, $R m$ stands for the number of ZR children at most and the maximum depth in the network is denoted by $\mathrm{Lm}$. The $\mathrm{Cm}$ and $\mathrm{Rm}$ are usually constants in the 
same ZigBee network. Every potential parent is given a finite sub-block of the address space, which is used to assign network addresses to its children. Given $\mathrm{Cm}, \mathrm{Lm}$, and $\mathrm{Rm}$, the assigned size of the address sub-block distributed by each parent at depth $d, C s k i p(d)$, is calculated as:

$$
C \operatorname{skip}(d)=\left\{\begin{array}{cr}
0 & R m=0 \\
1+C m \times(L m-d-1) & R m=1 \\
\left(1+C m-R m-C m \times R m^{L m-d-1}\right) /(1-R m) & R m>1
\end{array}\right.
$$

The network addresses $A_{d+1, r n}$ and $A_{d+1, e l}$ shall be assigned to the $n$-th router child and $l$-th end device child at depth $d+1$ in a sequential manner, respectively, as shown in (2):

$$
\begin{aligned}
& A_{d+1, r n}=A_{\text {parent }}+\operatorname{Cskip}(d) \cdot(n-1)+1 \\
& A_{d+1, e l}=A_{\text {parent }}+\operatorname{Cskip}(d) \cdot R m+l
\end{aligned}
$$

where $A_{\text {parent }}$ represents the address of the parent and $1 \leqslant n \leqslant R m$.

The hierarchical topology in ZigBee network is based on DAAM. In this tree shape structure, if the destination address is in the address space that a node is managing, the node forwards the packet to one of its child nodes. Otherwise, it forwards the packet to its parent.

\subsection{Neighbor table}

Based on the ZigBee specification, every ZigBee device maintains a neighbour table, which contains the information of all the nodes in its one-hop neighbourhood. Each neighbour entry should include the network's PAN (Personal Area Network) identifier; node's extended address, network address, device type, relationship, LQI and etc. Additional information such as the depth is also allowed to be added. Entries in the table are created/removed when the node joins/leaves to an existing network. Since the information on the neighbour table is updated every time a device receives any frame from a certain neighbour, the neighbour table can be regarded up-to-date all the time.

\section{Performance analysis for the ZigBee networks in 5G environment}

In this section, we move on to the performance analysis for the heterogeneous network which is composed of ZigBee and 5G devices. Since the 5G terminals which are able to join the ZigBee network have more sufficient resources, they have the potential to reduce the consumption of ZigBee nodes. Considering the different ways to utilize the $5 \mathrm{G}$ terminals, we develop two scenarios and are going to investigate them respectively.

\subsection{Two Scenarios}

One certain device may have one of the three roles (ZC, ZR or ZED) based on the ZigBee specification. To treat a $5 \mathrm{G}$ terminal as an end device is neither efficient nor meet the application requirements. Thus, we have 2 remaining options: to make $5 \mathrm{G}$ terminals work as coordinators or routers. We are going to dig up the performances in both scenarios.

\section{- Scenario 1 (S1): $5 \mathrm{G}$ terminals work as coordinators}

In this strategy, each 5G terminal, as a ZC, may initialize a unique network. Since the existing ZigBee nodes are not aware of the new network which works in a different channel, we 
develop a simple mechanism to make the $5 \mathrm{G}$ terminal "visible" for the ZigBee devices.

The 5G terminal acts its ZigBee role starting from the channel detection, during which it tries to find the existing ZigBee PAN and the available channel. If it finds a channel occupied, the channel information is restored. Before performing its ZC duty in a selected channel, the $5 \mathrm{G}$ device is required to announce its existence along with its working band in all the occupied channels by broadcasting a notification. When a ZigBee node receives the frame, it accordingly shifts its band after a random time with a preset expectation, and then inquires about its distributed address. The existing nodes decide to join the new networks if a network address with lower depth is provided. Afterwards, the recruit devices broadcast the notification with new PAN and channel information in their original bands. The joining decision making and announcing procedure repeats if needed so that the new PAN is visible to all the ZigBee devices around its coverage.

To fully explore the potential of the performance improvement, in this scenario, we allow a certain ZigBee node communicates in different PANs by in time channel switching with prior knowledge, which violates the ZigBee specification. The aim of this scheme is to keep the existing links in the initial ZigBee network and prevent the network segmentation caused by the disconnection when some nodes leave the current PAN [33].

By above settings, the scenario may result in a less topology distance to the $\mathrm{ZC}$ for some ZigBee nodes and the data transmission benefits from the lower ZTR hops.

\section{- Scenario 2 (S2): $5 \mathrm{G}$ terminals work as routers}

On the contrast, $5 \mathrm{G}$ nodes join the network as ZRs in this section. When successfully detected the existing ZigBee networks, they function as routers based on the acquired channel and PAN ID information. Note that this process does not instinctively improve the data transmission because the other ZigBee devices will not utilize the links involving the new participants until the network topology changes.

To make that happen, we also design a simple scheme. The 5G devices are asked to notify its existence and identities to its neighbors periodically. When a ZigBee unit receives the notification, it compares its current depth with the new one linked with 5G device. It updates its network address in the case of a lower depth can be obtained and announcing its address modification. The nodes in the neighborhood follow the same principle to decide whether they change their addresses [34]. By the explained method, the links via the 5G terminals may be used if it benefits the packet forwarding.

\subsection{Simulation results and analysis}

Table 1 Simulation Parameters

\begin{tabular}{ll}
\hline Simulation Parameters & Value \\
\hline Network Size & $100 \mathrm{~m} * 100 \mathrm{~m}$ \\
\hline Simulation Duration & $350 \mathrm{~s}$ \\
\hline Association Duration & $50 \mathrm{~s}$ \\
\hline Simulation times & 100 \\
\hline Number of ZigBee nodes & 200 \\
\hline \multicolumn{1}{c}{ Deployment } & Random (Uniform) \\
\hline Location of ZC & Center \\
\hline $\mathrm{Cm} / \mathrm{Lm}$ & $4 / 7$ \\
\hline
\end{tabular}




\begin{tabular}{cl}
\hline \multicolumn{1}{c}{ Transmission Range } & $25 \mathrm{~m}$ \\
\hline Propagation & Two-ray \\
\hline Data packet size & $1 \mathrm{~KB}$ \\
\hline Packet interval & 1 packet/s \\
\hline Packet start time & $50-100 \mathrm{~s}$ (Uniform) \\
\hline Destination address & Random \\
\hline Number of 5G nodes & $0,5,10,15, \ldots, 50$ \\
\hline First appearance & $50-100 \mathrm{~s}$ (Uniform) \\
\hline Trajectory & Straight line \\
\hline Average speed & $1.5,5,10,15,20 \mathrm{~m} / \mathrm{s}$ \\
\hline
\end{tabular}

Based on the mentioned designs, the simulations on different scenarios of ZigBee networks with 5G devices are carried out to evaluate their performances. The general network settings for NS-3 are shown in Table 1. To analyze the effect of the number of the joining $5 \mathrm{G}$ nodes, the number of exotic devices varies from 0 to 50 (step by 5). The standard ZigBee configuration in which the 5G devices only join the ZigBee network as determined device type without any further functions is compared with the $\mathrm{S} 1$ and $\mathrm{S} 2$ as a reference. To emulate the actual ZigBee application, the destination address is randomly chosen. The packet is transmitted to the $\mathrm{ZC}$ in $50 \%$ chance, and in another $50 \%$, the destination is selected from other devices in equal probability. Moreover, the packet interval for the $\mathrm{ZC}$ is 10 packets per second. The mentioned 3 types of frames stand for the sensing data, the general communication and the command and control packets respectively. Considering the mobility of $5 \mathrm{G}$ nodes, the following mechanism is adopted. Each $5 \mathrm{G}$ device begin to take part in the simulation at a random time from 50 to 100 seconds after the simulation starts. It firstly appears at a random position on the boundary of the simulation square. The initial moving direction is going into the simulation area and the trajectory is a straight line. In our first simulations, the average speed is $1.5 \mathrm{~m} / \mathrm{s}$ which is approximately the walking speed of an adult. When reaching the boundary, the $5 \mathrm{G}$ node has a fifty-fifty chance to reflect the trajectory with the same entry and exit angles or escape from the simulation area. In the latter case, a new 5G node appears at a random position on the boundary and act as the mentioned procedure.

We firstly investigate the network performance including the packet delivery ratio, average hop counts and the end-to-end delay, which are shown in Fig.2. Generally speaking, compared with the standard ZigBee specification, both the two scenarios improve the data transmission as the number of 5G nodes increases and S1 outperforms S2. As shown in Fig.2 (a), the packet delivery ratio degenerates as more nodes participate in the network. However, the PDR of S1 and S2 decrease slower and have a similar performance. As to the hop counts in Fig.2(b), because the 5G nodes may not optimize the transmission link based on the ZigBee specification, the hop counts for standard ZigBee remain a similar level, and the updating mechanisms in S1 and S2 enable the topology updating and result in less hops. Since we focus on the performance of ZigBee devices, only the transmission starts from a ZigBee node is counted (which means that the data sending from a $5 \mathrm{G}$ terminal is excluded in the hop counting). S2 slightly reduce the hop counts while S1 has a greater effect. Although the packets sent by ZigBee nodes decrease, the total hop counts including the 5G nodes retransmission do not change much. This result in the similar end-to-end delay in Fig.2 (c). The higher latency in S1 and S2 is mainly because the bandwidth occupation caused by the introduced topology maintenances. In the case of $505 \mathrm{G}$ nodes, the PDR 
of S1 and S2 are $9.04 \%$ and $7.92 \%$ better, the hop counts are $16.36 \%$ and $4.88 \%$ less and the end-to-end delay are $2.92 \%$ and $0.93 \%$ higher than the standard ZigBee network.

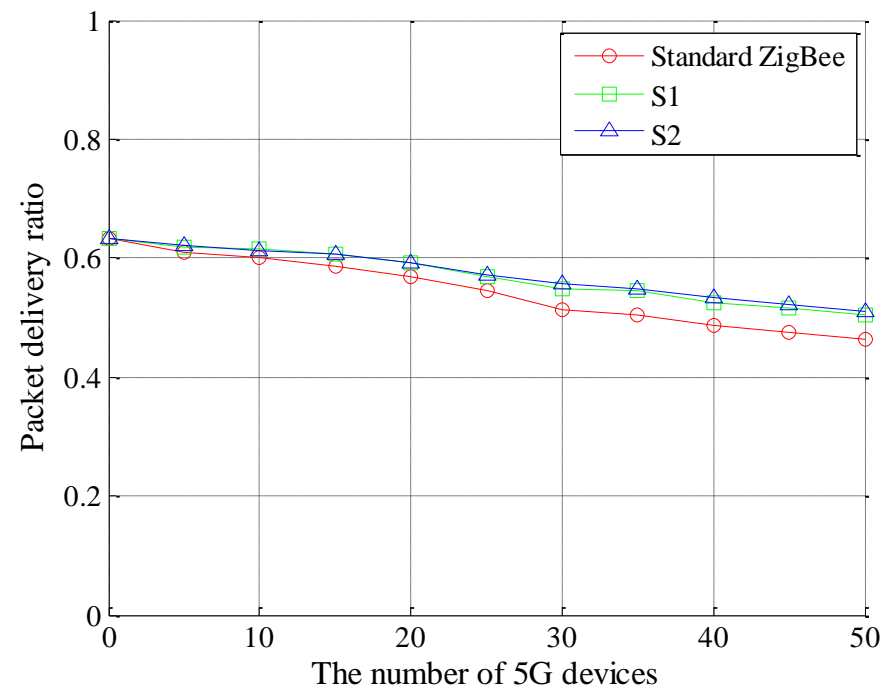

(a)

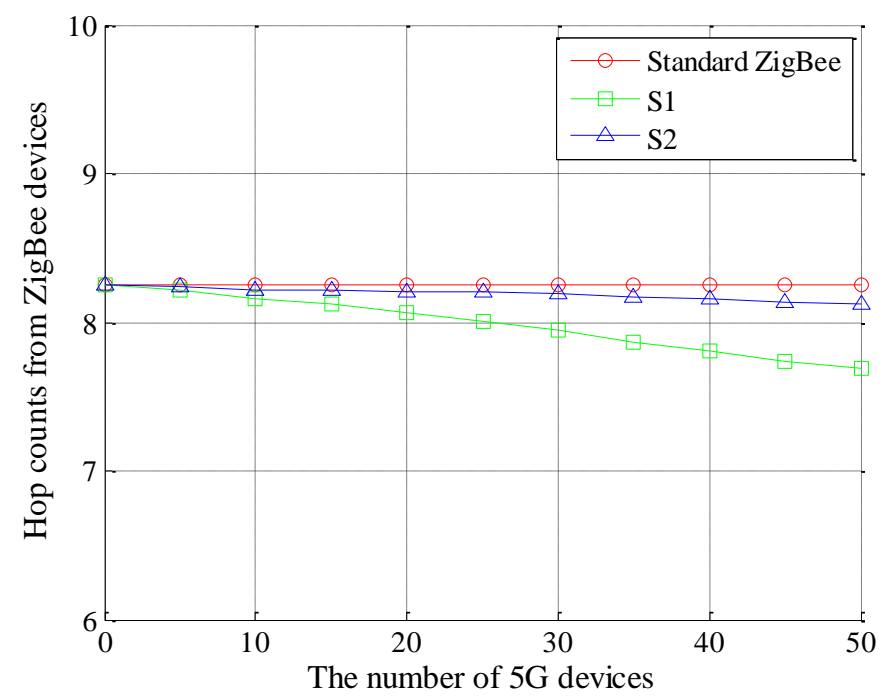

(b) 


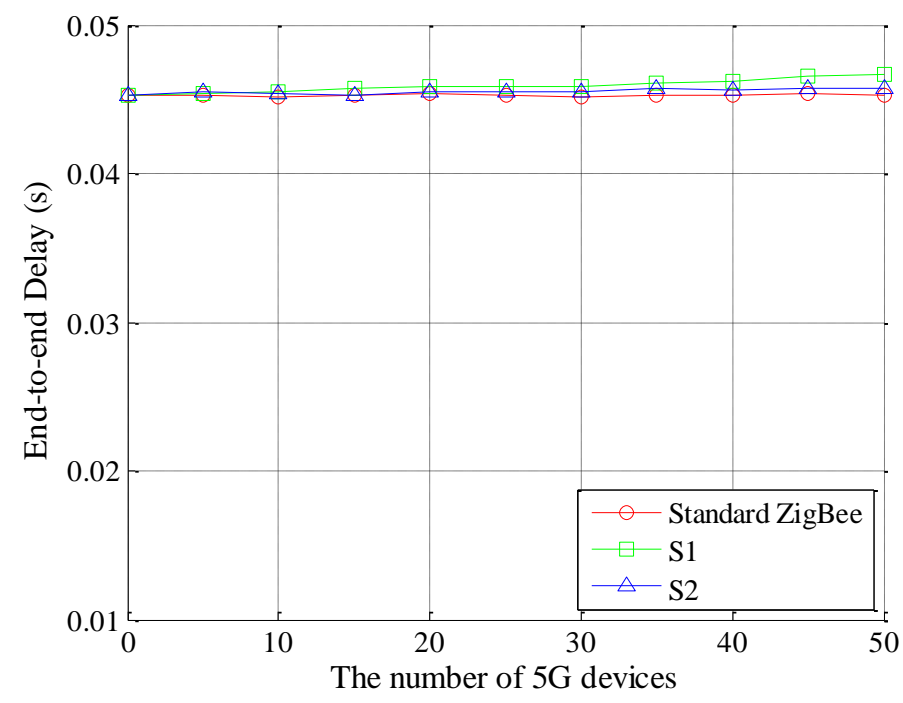

(c)

Fig.2. Performance evaluation for different scenarios

(a) packet delivery ratio (b) hop counts (c) end-to-end delay

The network performances show that our scenarios are capable of improving the ZigBee data transmission in 5G environment. However, to make a comprehensive analysis, the costs of different deployments are also discussed. The packets sent per ZigBee node and the normalized routing overheads are studied as shown in Fig.3. Note that both the data and command packets successfully sent by ZigBee nodes are counted and the number can reflect the network traffic and the bandwidth occupation. Since the topology has little change in the standard ZigBee networks, its value almost keeps the same in Fig.3 (a). On the other hand, S1 and S2 both have more packets owing to their efforts to optimize the paths, and the co-existence of different PANs in S1 leads to a much higher consumption for ZigBee devices. Technically speaking, the ZTR has no routing overheads because only the parent-child links based on the DAAM are used. Whereas the topology updating may introduce extra communication, the related frames are regarded as the overheads for the improved links. Fig.3(b) indicates that the more 5G terminals are in the networks, the more cost S1 and S2 may have, and the S1 has much higher overheads because the 5G devices are the ZCs with 0 depth in $\mathrm{S} 1$ and all the nodes in a certain PAN are involved in the topology maintenances. On the contrary, the router role of 5G nodes in $\mathrm{S} 2$ only brings about local communication. In the networks with $505 \mathrm{G}$ terminals, each ZigBee nodes send $27.11 \%$ and $7.93 \%$ more packets in S1 and S2, and the normalized routing overheads are $37.02 \%$ and $10.30 \%$. 


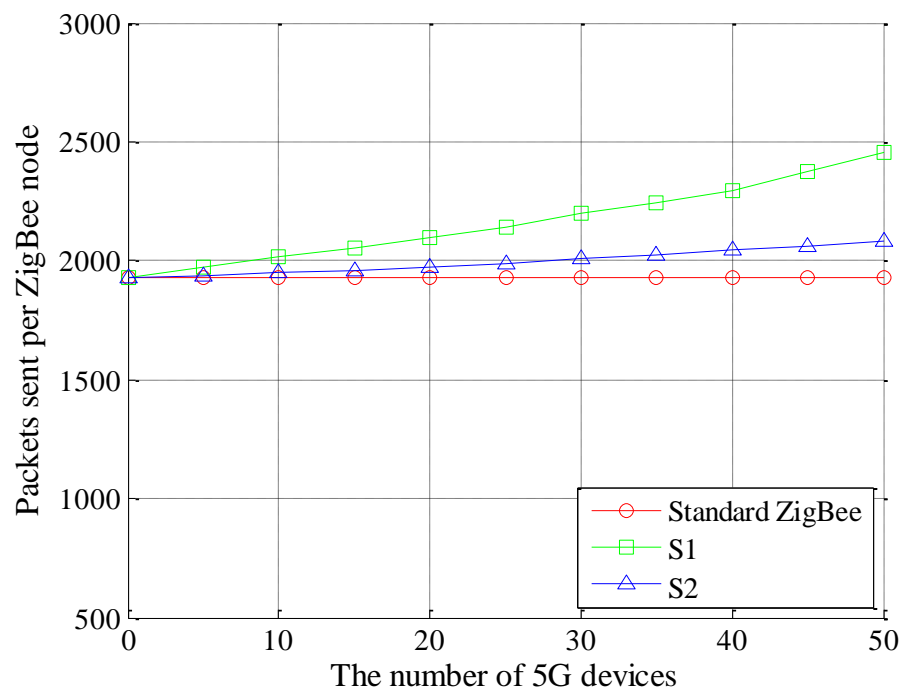

(a)

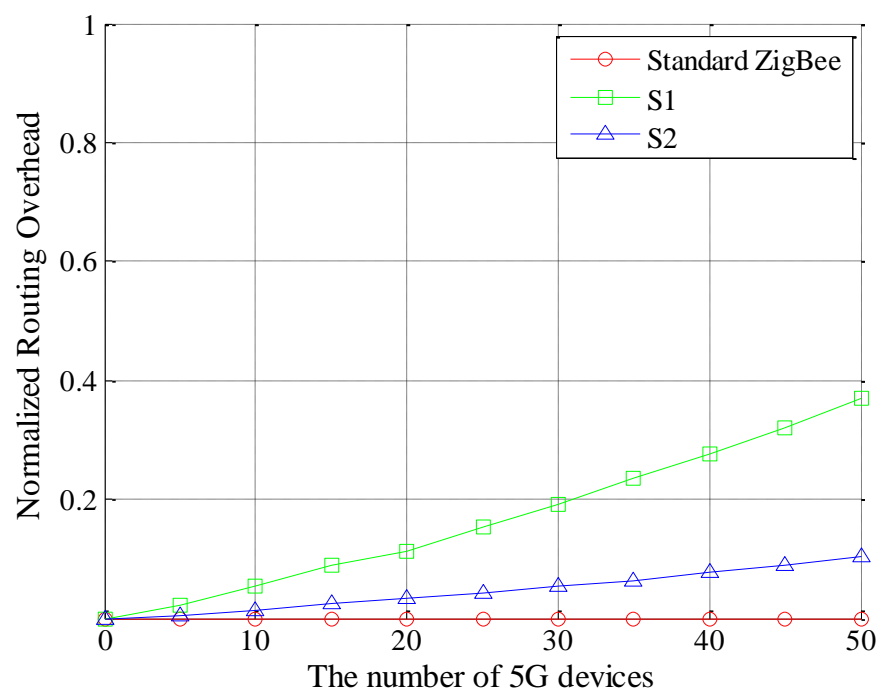

(b)

Fig.3. The overhead for different scenarios

(a) packets sent per ZigBee node (b) normalized routing overhead

Although the improvement is limited because the $5 \mathrm{G}$ devices only function as common ZigBee devices and their abundant resources are not exploited. The performance analysis shows that our scenarios improve the data transmission in ZigBee networks. S1 and S2 both have better PDRs and less hop counts. On the other hand, these two scenarios also introduce more traffic and the routing overheads. The multiple ZCs in S1 lead to greater improvement as well as more costs than S2. Besides the overhead may be too large for ZigBee devices, some other issues also make S1 hard to realize. Firstly, as mentioned, a ZigBee device cannot work in different bands simultaneously. The channel switching seems like a rational solution, but the cycling interval is impossible to precisely determined, to say nothing of the related energy consumption. Secondly, the co-existence of different networks may introduce unnecessary inter-channel interference (ICI). Especially in the case of a large amount of $5 \mathrm{G}$ terminals, the most legal channels are occupied and 
the ICI tends to increase. Finally and most importantly, one of the inherent features of $5 \mathrm{G}$ device is its mobility. In our previous simulations, the 5G device mobility is low; Fig. 4 shows the performances of different scenarios with growing mobility of $5 \mathrm{G}$ devices (The number of $5 \mathrm{G}$ devices is fixed 50). It can be seen that the performance of S1 quickly falls and the overheads are also increasing rapidly. And S2 is less sensitive to the changing of the moving speed. In the case of the $20 \mathrm{~m} / \mathrm{s}$ average speed (the approximate speed of moving vehicles), $\mathrm{S} 2$ has a similar PDR as the standard ZigBee while the performance of S1 is about $12.93 \%$ worse. The hop counts of 3 scenarios tend to be equal, that is mainly because the high mobility reduces the duration of better links. The moving 5G terminal may be within the coverage of the ZigBee networks for only a short while; such an ephemeral network lifetime may extremely declines the benefit. Since the ZC is always regarded fixed in the ZigBee network, the mobile ZC may bring about the frequently changes of the network address for its descendants, the latency, packets sent per ZigBee nodes and the routing overhead are all increasing as the mobility adds, and the results for $\mathrm{S} 1$ are much worse than S2. They are unaffordable overheads for the ZigBee nodes with limited resources.

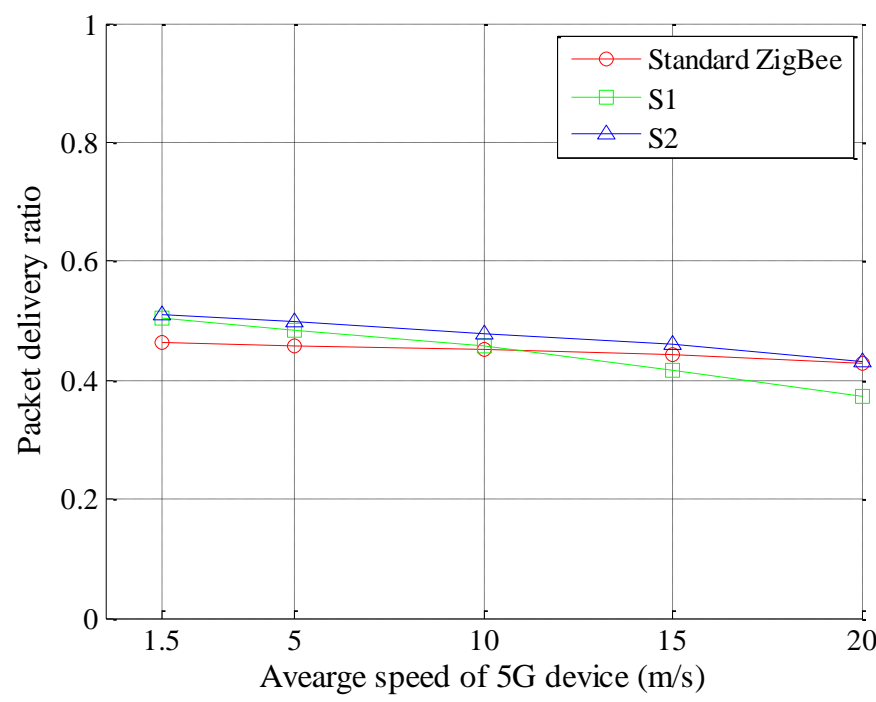

(a)

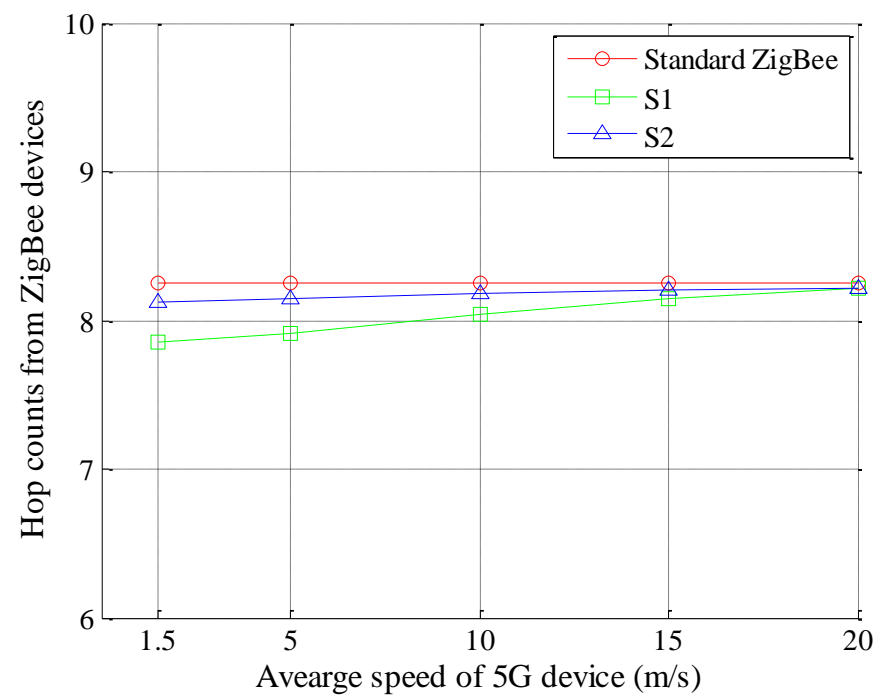

(b) 


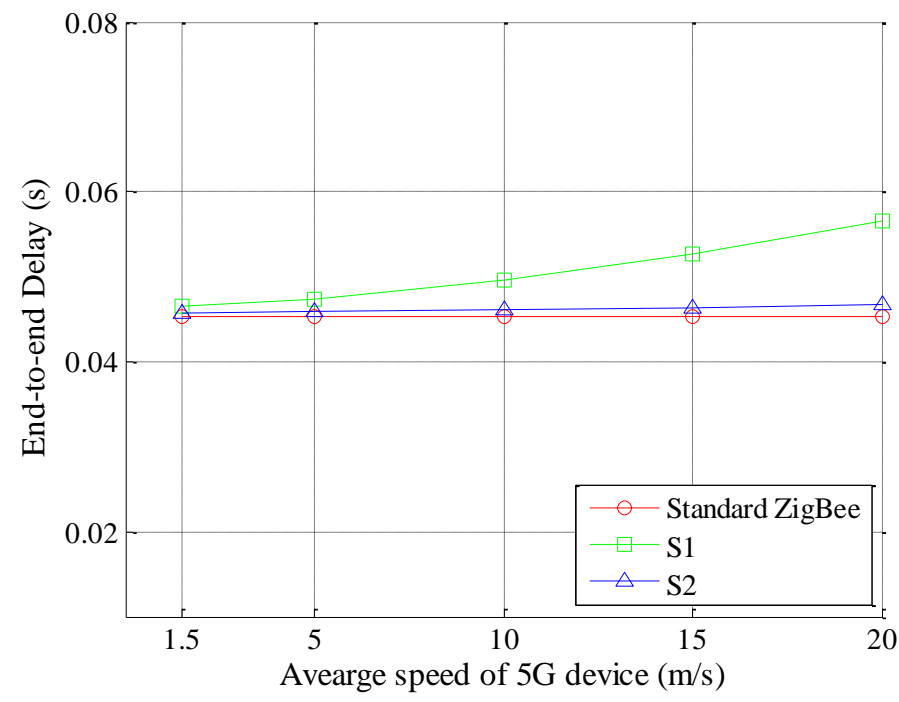

(c)

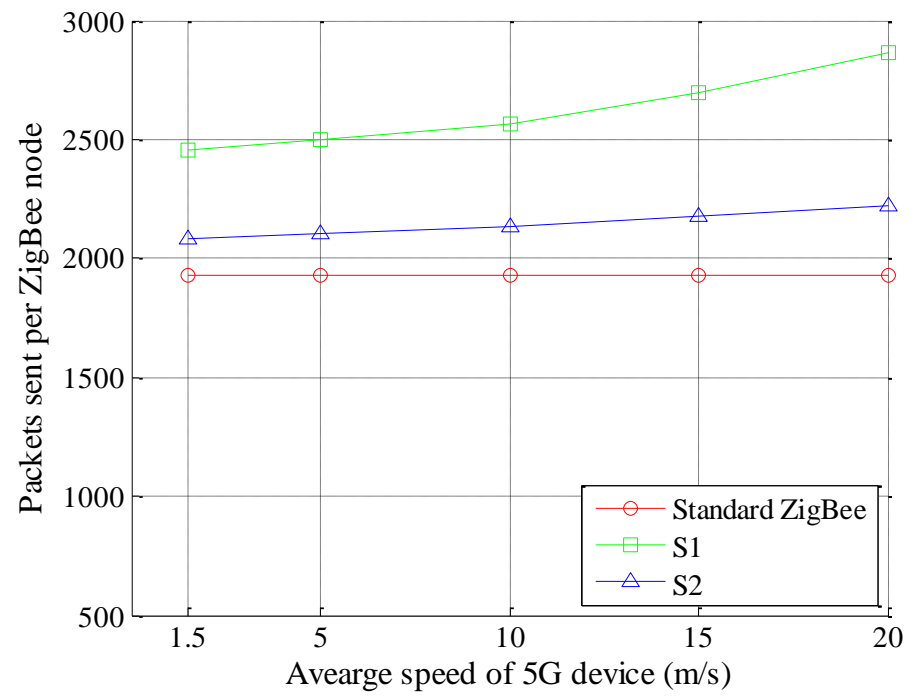

(d)

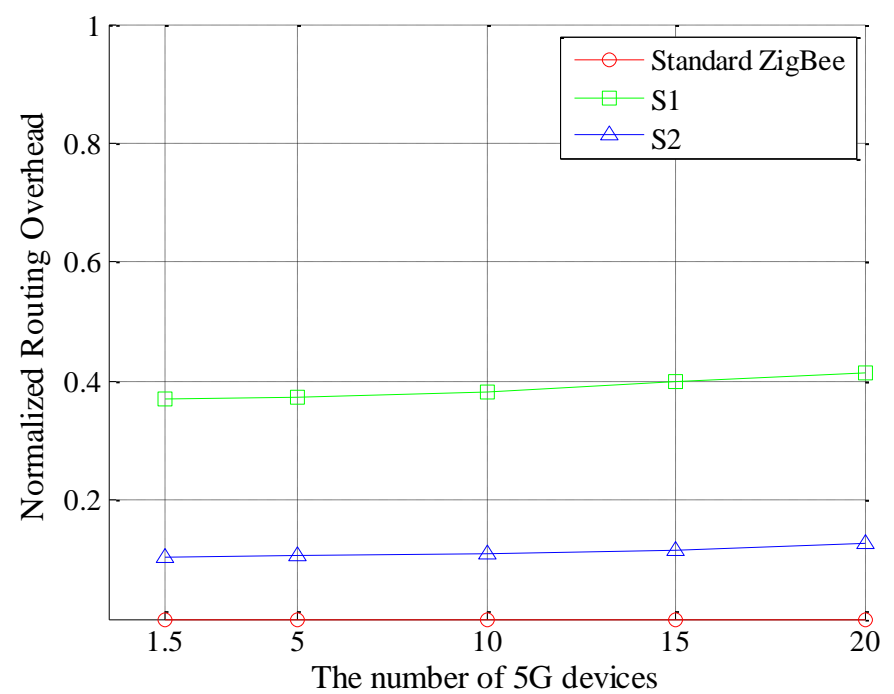


(e)

Fig.4. Performance evaluation for different scenarios with different $5 \mathrm{G}$ node mobility (a) packet delivery ratio (b) hop counts (c) end-to-end delay (d) packets sent per ZigBee node (e) normalized routing overhead

Based on the above analysis, S1 is not a practical solution for the heterogeneous networks. Despite of its drawbacks, $\mathrm{S} 1$ brings us the inspiration to treat the $5 \mathrm{G}$ devices as 0 depth nodes to optimize the path. However, based on the ZigBee specification, the ZC is the only 0 depth device and the ZTR follows the hierarchy which determines the depth. To fully take advantage of the resources in $5 \mathrm{G}$ devices, in the next section, the improved routing algorithm is developed to break the limitation of parent-child links in the ZTR based on the framework of S2.

\section{Nearest Access Routing for the ZigBee network in 5G environment}

To improve the data transmission in the ZigBee networks in the $5 \mathrm{G}$ environment, the key is to make use of the cellular resources as much as possible. This effort can be divided into 2 tasks: to discover the $5 \mathrm{G}$ devices and the routing via them.

\subsection{Maintenance of physical depth}

We introduce the concept of physical depth (PD) to achieve our goal. The depth in the multi-hop networks is usually defined as the hops from the local device to the sink node based on a certain routing method. The ZTR is the reference in defining the existing topology depth (TD) in the ZigBee network. The measure indicates the hop counts via the parent-child links. On the contrary, the introduced physical depth is defined as the minimum hops to a node which is able to access the IP network.

In our algorithm, the physical IP depth values of all the ZigBee devices are set -1 for initial. And the $5 \mathrm{G}$ terminal is tagged for recognition. The $\mathrm{PD}$ is fixed 0 for the $5 \mathrm{G}$ terminal and the $\mathrm{ZC}$. To be distinguished from the topology depth, the following method is adopted for the PD updating We make the PD be one of the required entries in the neighbor table. The PD for the current device is one plus the minimum non-negative PD value in its neighbor table. Since the neighbor table updating can be regarded up-to-date, this strategy guarantees the real time PD maintenance. For example, a ZigBee node in the neighborhood of a joining 5G terminal may set its PD 1 because of the PD 0 entry of the 5G node in its neighbor table, and the PDs of its neighbor nodes will be no more than 2. Considering the case of 5G terminal leaving, if the PD of a device is set 1 owing to a tagged $5 \mathrm{G}$ device, the node resets its PD to -1 when the corresponding entry is no more available in its neighbor table.

By this principle, the PDs of the devices in the network are effectively managed. Since the PDs of nodes in the neighborhood of $5 \mathrm{G}$ terminals are determined as 1, the PD maintaining mechanism instinctively solve the discovery problem by the PD change of ZigBee devices.

\subsection{Nearest access routing algorithm}

Now we work on the routing issue. Though the $5 \mathrm{G}$ cellular and the mobile internet are utilized to share the loads in the ZigBee network, we assume that the data in the IP network can always be successfully transmitted and the IP routing method is beyond our work in this paper. We 
focus on the routing improvement in the ZigBee networks. The nearest access routing (NAR) algorithms for different communication types are severally discussed.

\section{- Uplink packet (many-to-one)}

Technically, the uplink and downlink refer to the transmission to and from the base station in the cellular network. In this paper, we use the terms to describe the communication between a ZigBee device and the sink node. The sensing data gathered by the sink follows the uplink, while the feedback and command frames from the sink is transmitted in downlink.

The routing for the uplink data is quite simple. When a node is sending a packet, the next hop destination is chosen among its neighbors with the minimum PD. If there are several devices have the same minimum PD, the one with the best link quality is selected. Note that because the link quality is the primal consideration in the ZigBee hierarchy forming, the parent-child link keeps the first priority if it provides the least remaining hops.

\section{- Downlink packet (one-to-many)}

In NAR, every $5 \mathrm{G}$ terminal participated in the ZigBee network is required to upload its neighbor table and routing table entries to the ZC. By gathering and updating the reported information, a shared neighbour table and routing table are maintained and each $5 \mathrm{G}$ device is able to access the tables via internet. Because the information exchange is carried out in IP networks, we regard the procedure has no costs for ZigBee devices.

When a downlink packet is sending, all the nodes in the shared neighbour table are candidates for the one hop destination. If the frame is sent to one of the candidates, it is directly transmitted. Otherwise, the candidate with the minimum hop counts to the destination in ZTR is selected to receive the data, and then it forwards the frame to the destination by ZTR.

To decide the ZTR hops between two given network addresses, the following algorithm based on DAAM is employed. Given the network address $A d d r_{x}$ of a node $X$, the Algorithm 1 decides its topology depth $T D_{x}$.

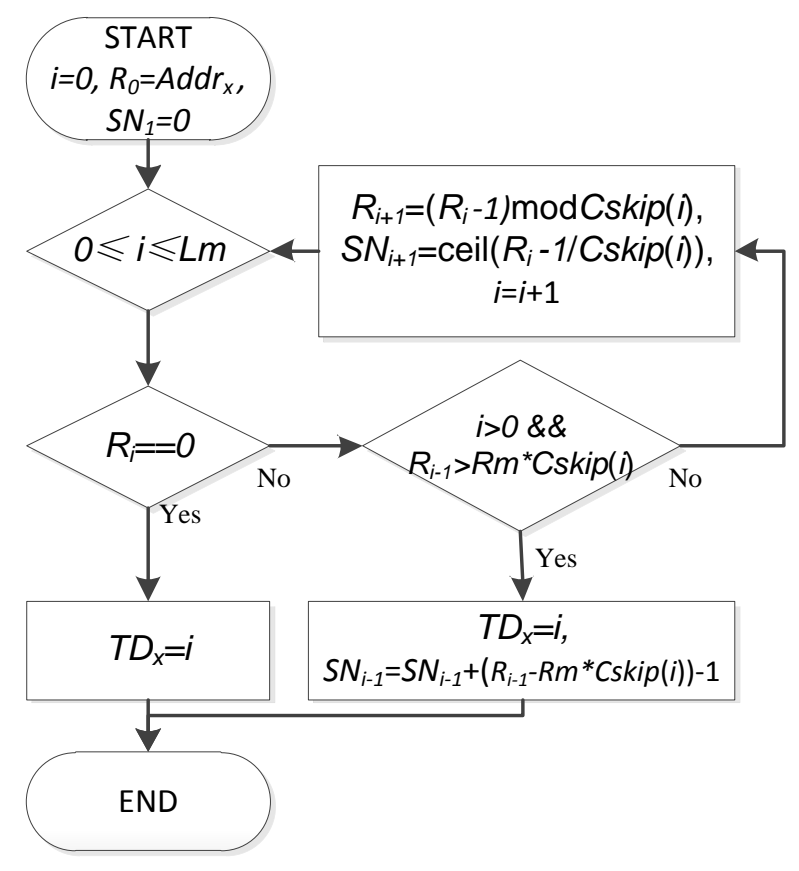

Fig.5. The Diagram of topology depth deciding 
In the algorithm, along with the topology depth, we also obtain a vector $S N=\left[S N_{1}, S N_{2}, \ldots\right]$. For a node in topology depth $T D_{x}$, the previous $T D_{x}$ elements in $S N$ are non-zero. And the $S N_{i}$ value indicates the sequential number of the node or the node's ancestor in depth $i$. Given the $S N$, the network address of the node's ancestor in depth $i$ can be calculated as:

$$
\begin{aligned}
& \text { AnceAddr }(i)=\left(1+S N_{1} \cdot \operatorname{Cskip}(0)\right)+ \\
& \left(1+S N_{2} \cdot \operatorname{Cskip}(1)\right)+\cdots+\left(1+S N_{i} \cdot \operatorname{Cskip}(i-1)\right) \\
& =\sum_{k=1}^{i} S N_{k} \cdot \operatorname{Cskip}(k)+i
\end{aligned}
$$

For two nodes $X$ and $Y$, the lowest common ancestor (LCA) is defined as the common ancestor for $X$ and $Y$ with the largest depth. Given the addresses of $X$ and $Y$, based on the Algorithm 1, the $T D_{x}, T D_{y}, S N_{x}$ and $S N_{y}$ can be obtained. Compare the corresponding elements in $S N_{x}$ and $S N_{y}$, starting from the first pair $S N_{x l}$ and $S N_{y l}$. If they are not equal, it can be concluded that the LCA for $X$ and $Y$ is the ZC with 0 topology depth. Otherwise, the next pair is compared. The comparing keeps going as the sequential number $k$ increases until one different pair $S N_{x k}$ and $S N_{y k}$ is found. On this occasion, the depth of the LCA, $T D_{L C A}(X, Y)$ is $k-1$ and its address can be calculated by Equation (1) with the first $k-1$ same sequential numbers in $S N_{x}$ and $S N_{y}$.

Finally, the hop counts from node $\mathrm{X}$ to $\mathrm{Y}$ in ZTR $H_{Z T R}(X, Y)$ can be calculated as:

$$
H_{Z T R}(X, Y)=T D_{x}+T D_{y}-2 T D_{L C A}(X, Y)
$$

When the ZC sends a packet to a device via downlink, for each candidate in the gathered neighbor table entry, it calculates the $H_{Z T R}(X, Y)$ be setting the destination $X$ and the candidate $Y$. By IP networks, the frame is sent to the $5 \mathrm{G}$ device (or the $\mathrm{ZC}$ itself), whose neighbor table includes the candidate with the minimum $H_{Z T R}(X, Y)$ value. If several devices share the same minimum hop counts, the one with be best link quality is chosen. And then the data is transmitted to the destination in ZigBee network.

\section{- General communication (any-to-any)}

The general communication describes the transmission from a general device to another one, during which the $\mathrm{ZC}$ is neither the source nor the destination. When it happens, the sending device has to firstly decide to transmit the packet in ZTR or with the assistance of $5 \mathrm{G}$ terminals. If the sending node is $X$ and the receiving one is $Y$, the hop counts via 5G terminals, $H_{N A R}(X, Y)$, can be obtained by (3).

$$
H_{N A R}(X, Y)=P D_{x}+P D_{y}
$$

Since a general device is not aware of the gathered neighbor table or the PD of $Y$. The exact transmission hops via 5G terminals cannot be accurately predicted. However, it is obvious that the transmission passes through the $\mathrm{ZC}$ for the worst case. Thus the upper bound for the $H_{N A R}(X, Y)$ is shown as:

$$
H_{N A R}(X, Y) \leq P D_{x}+T D_{y}
$$

Thus, based on (2) and (3), to compare the differences between $H_{N A R}(X, Y)$ and $H_{Z T R}(X, Y)$ :

$$
H_{N A R}(X, Y)-H_{Z T R}(X, Y)=\left(P D_{x}-T D_{x}\right)+\left(P D_{y}-T D_{y}\right)+2 T D_{L C A}(X, Y)
$$

If the result in formula (5) is no more than 0 , it means that the NAR could provide a routing 
path no longer than the ZTR. Since the value of $\left(P D_{y}-T D_{y}\right)$ is not accessible, we have to estimate the value of this term. One method is to assume the symmetry in the network, since the random distributed 5G nodes may identically affect the topology for each node, it is rational to estimate the $\left(P D_{y}-T D_{y}\right)$ is equal to $\left(P D_{x}-T D_{x}\right)$. On that occasion, the equation (5) can be written as:

$$
T D_{x}-P D_{x} \leq T D_{L C A}(X, Y)
$$

Thus, in NAR algorithm, the sending device may check whether the parameter values satisfy inequality (6). If not, the source node directly forwards the packet to the destination in ZTR links. Otherwise, the data is to be sent to the nearest $5 \mathrm{G}$ node by the mechanism described in the uplink transmission. Among the candidates in the gathered neighbor tables, the packet is further transmitted to the node with the minimum hop counts in ZTR based on equation (2). And then the frame is sent to the destination by ZTR.

Based on the previous discussion, the NAR is able to make use of the $5 \mathrm{G}$ devices to optimize the transmission path in ZigBee networks without introducing high overheads. An example is demonstrated in Fig.6. If the ZigBee node $\mathrm{G}$ is communicating with the $\mathrm{ZC}$, since its neighbour $\mathrm{F}$ has the lowest PD value 1 in its transmission range, the uplink follows G-F-1-ZC. Compared with the standard ZigBee link G-D-B-A-ZC whose hop counts is 4, the total counts for NAR is 3 . However, the hop counts from ZigBee nodes are 2, since they only send 2 packets ( $G$ and F). For the downlink, because we minimize the extra communication for ZigBee devices, node 1 is not aware of the optimum path from F to G. Among all the candidates in the shared neighbour table, which are E,F,A,H and J, since the E has the least remaining ZTR hop counts, it is selected as the next hop node and the whole path is ZC-I-E-D-G. The ZigBee devices forward 2 frames in total, while the standard ZTR has 4 hops. The transmission from G to $\mathrm{I}$ is a case for the general communication, the whole path is G-F-1-2-M-J-I and I-J-2-1-E-D-G, the hops from ZigBee nodes are 3 and 4, the hop counts for ZTR is 6 . Note that when 1 receives packets from F and chooses the next hop among candidates ZC and node 2, although they have the same remaining ZTR hops which are 2, the node 2 is preferred in NAR because it may alleviate the congestion in low depth node $\mathrm{H}$.

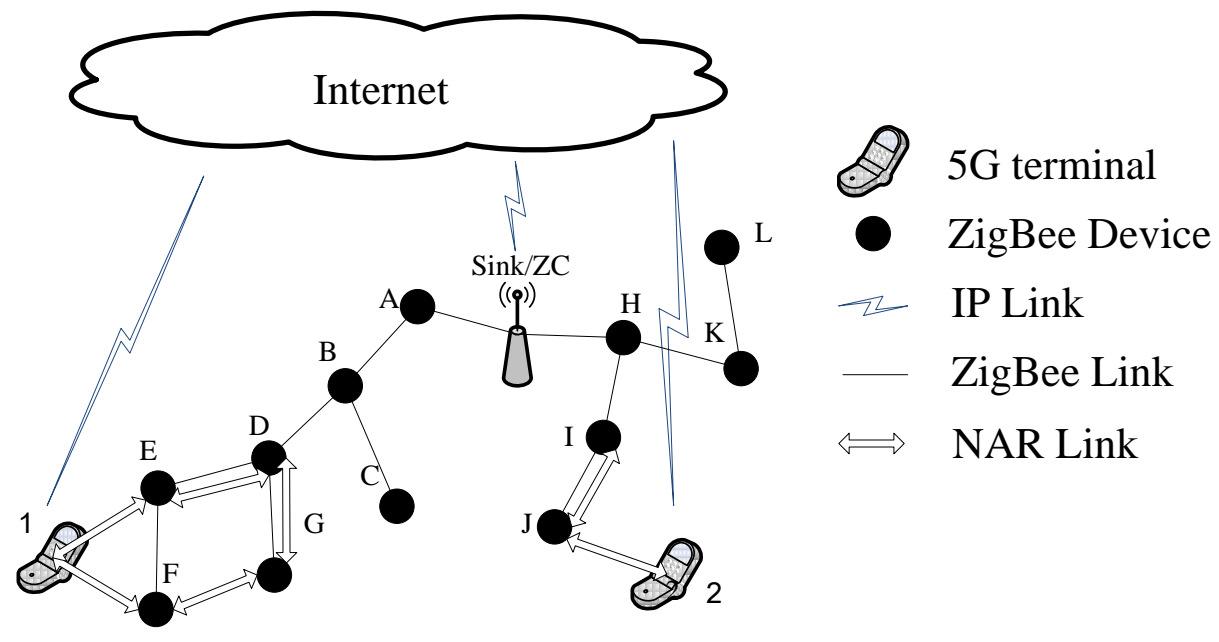

Fig.6. An example of the NAR 


\section{Simulation}

In this section, we evaluate the performances for the NAR in diverse metrics of PDR, hop counts (from ZigBee devices), end-to-end delay, packets sent per ZigBee node and the normalized routing overhead as in Section 4. We keep the same general settings in Table 1, and the NAR, S1, S2 and standard ZigBee networks are compared.

The performance of NAR with different numbers of 5G devices is shown in Fig.7. The PDR in Fig.7 (a) indicates that the PDR of NAR does not change much as the density of 5G nodes adds. On one hand, the optimized path may benefit the PDR by less hop counts and more diverse route; on the other hand, the disconnection cause by node movement .And these two aspects tend to cancel each other in our simulation. Fig.7 (b) demonstrates the most significant improvement by NAR, the hop counts from ZigBee devices. This value is reduced $43.89 \%$ for the best case. The end-to-end delay is also improved as in Fig.7(c). The lower latency mainly results from the shorter hop counts in total. However, the transmission from $5 \mathrm{G}$ device needs to be covered, the improvement is not as much as the hop counts, about 19.65\%. Another advantage of NAR is its low cost, as illustrated in Fig.7 (d) and Fig. 7(e). Different from S1 and S2, NAR is able to lower the packets sent per ZigBee node compare with the standard ZigBee, because the IP links are utilized for the communication between different 5G devices (and ZC). In the network with 50 5G devices, each ZigBee node sends $17.88 \%$ less frames. Moreover, since NAR is based on the PD maintenance in which only 5G devices broadcast their appearances and the PD diffusion is based on the neighbour table updating, the overhead of NAR for ZigBee nodes are 0. In summary, NAR achieves better performance with lower costs; the more 5G nodes take part in the ZigBee network, the bigger improvements it makes.

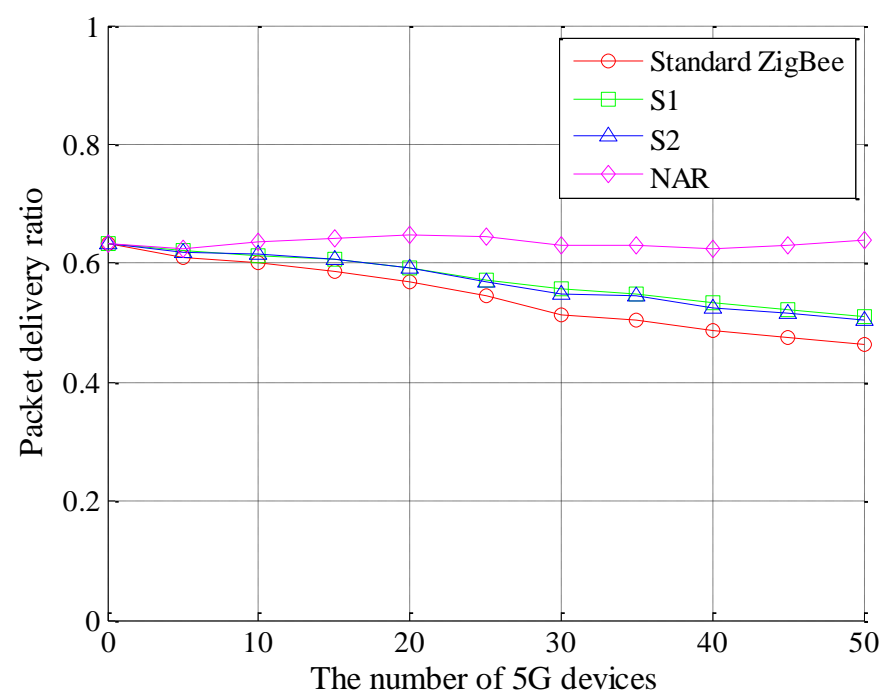

(a) 


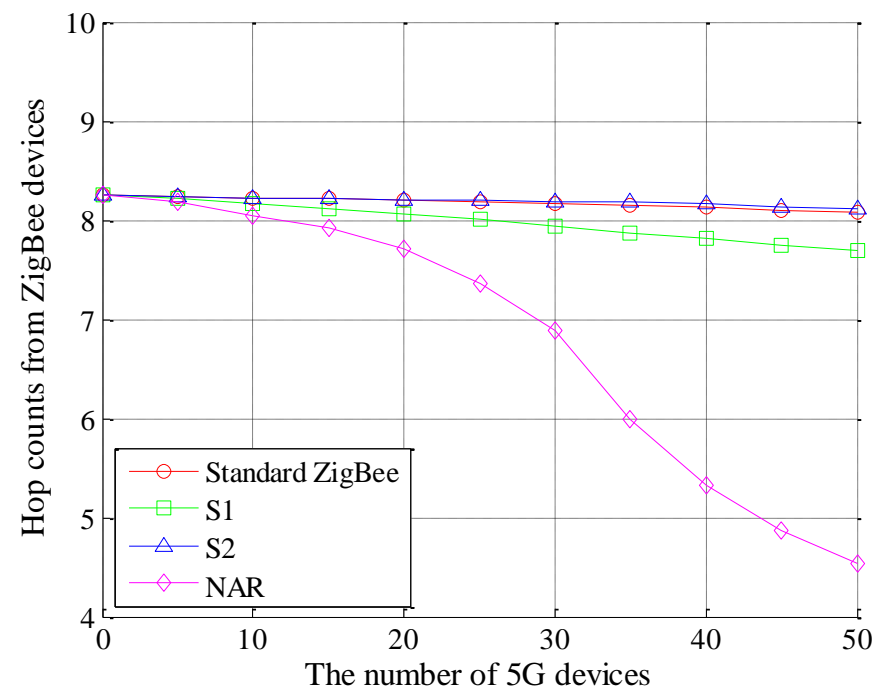

(b)

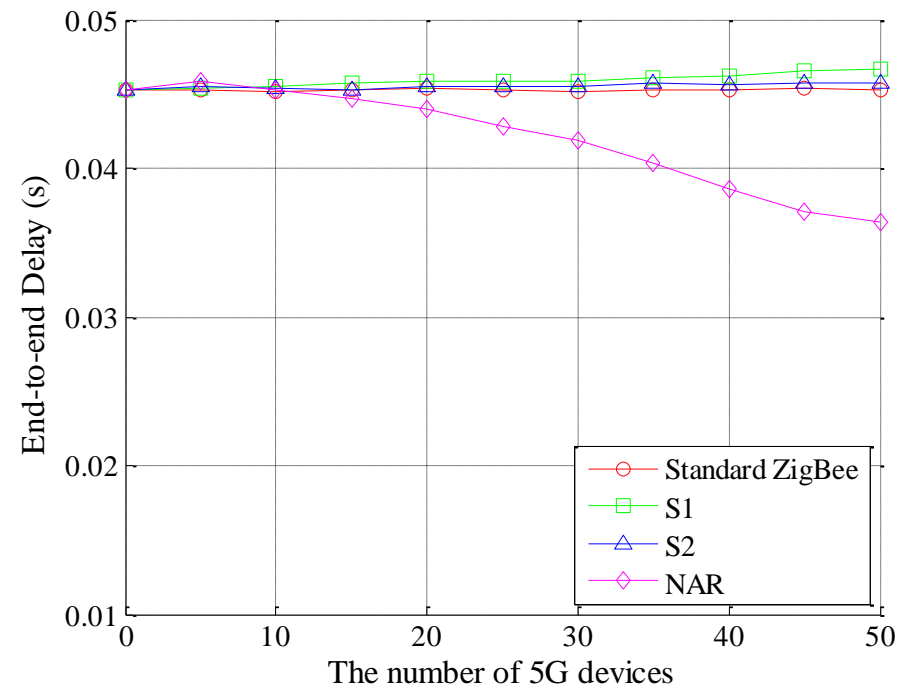

(c)

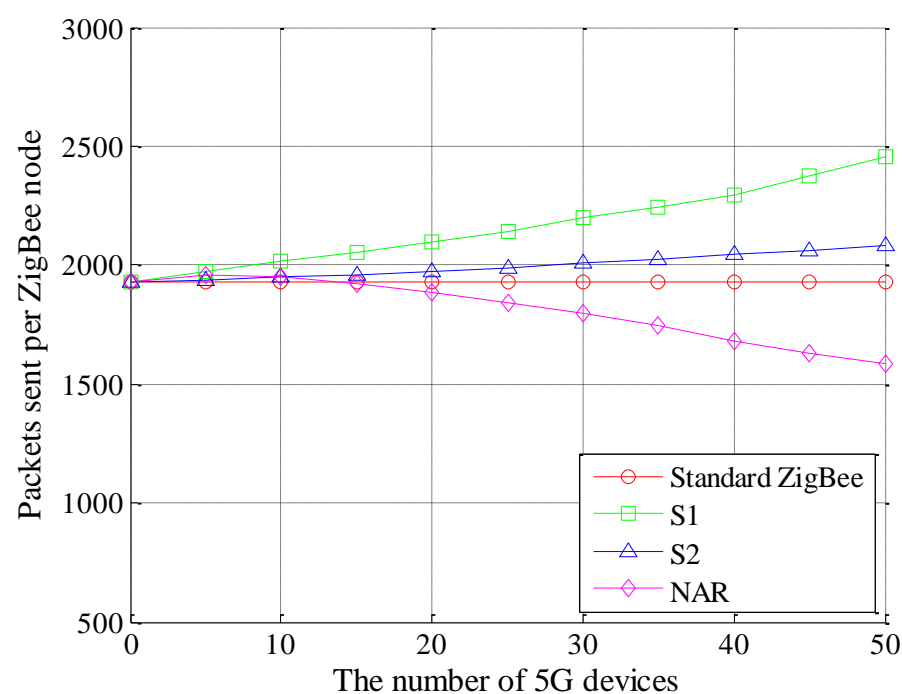




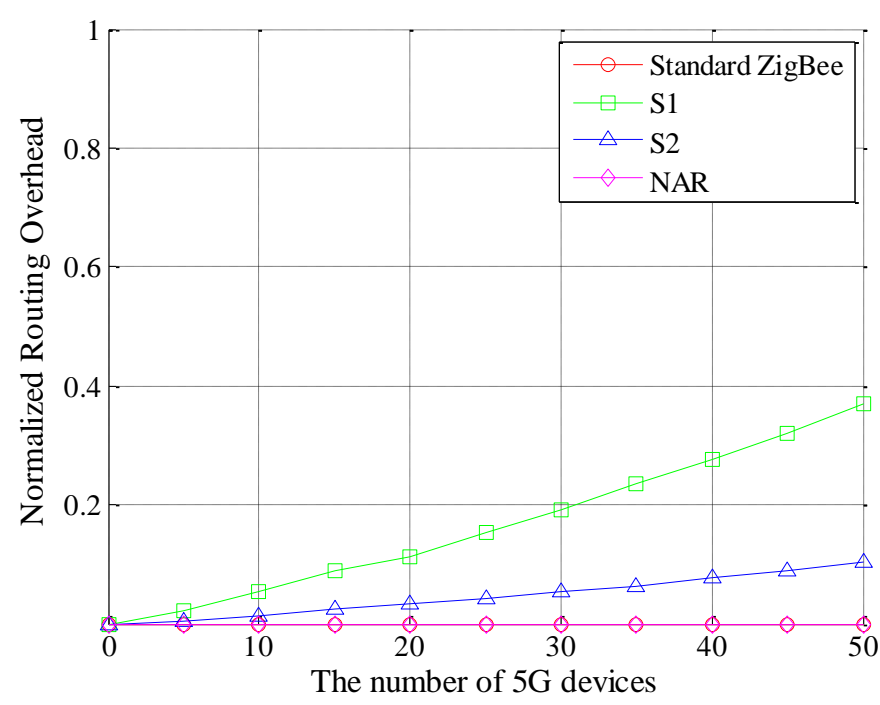

(e)

Fig.7. Performance evaluation for different scenarios with different numbers of $5 \mathrm{G}$ devices (a) packet delivery ratio (b) hop counts (c) end-to-end delay (d) packets sent per ZigBee node (e) normalized routing overhead

The effect of $5 \mathrm{G}$ nodes mobility is considered and tested as well. By varying the average speed as in Section 4, the results are figured in Fig.8. Overall, NAR is more sensitive to the mobility, the main reason is that the $5 \mathrm{G}$ devices actively gather the packets in their neighborhoods, so the high mobility leads to the more frequently changes of the optimized paths. The PDR falls fast as the node average speed rises up in Fig.8 (a). For the worst case, the PDR deteriorates worse than S2 and standard ZigBee. Meanwhile, the improvement on the hop counts also degenerates, for the highest mobility in Fig.8 (b), this value is only 9.79\% better than standard ZigBee. The same to the PDR, the latency which is shown in Fig.8 (c) is also possible to have poorer performance than S2 and standard ZigBee in the case of $20 \mathrm{~m} / \mathrm{s}$ node speed. Since the normalized routing overheads remain zero, we only show the packets sent per ZigBee node for the routing costs. It can be seen in Fig.8 (d) that the number of packets tends to be close to the level of standard ZigBee as the nodes mobility increases.

Based on the given results, when the mobility of $5 \mathrm{G}$ devices is not so high, NAR is able to significantly improve the performances for the ZigBee networks. The high PDR guarantee the efficiency in communication. The less hop counts, fewer packets and zero overheads for ZigBee nodes may lead to less energy consumption which is one of the most important targets for ZigBee networks. The shorter delay is another advantage for NAR though the latency is not as crucial as the mentioned factors,. The improvement on both the performances and overheads means the $5 \mathrm{G}$ devices successfully share the communication for ZigBee networks in the proposed scheme. Although the improvement declines as the 5G node mobility increases, NAR still save the consumption for ZigBee devices thanks to fewer packets sent, while the other metrics are at least remain similar levels as the standard ZigBee networks. 


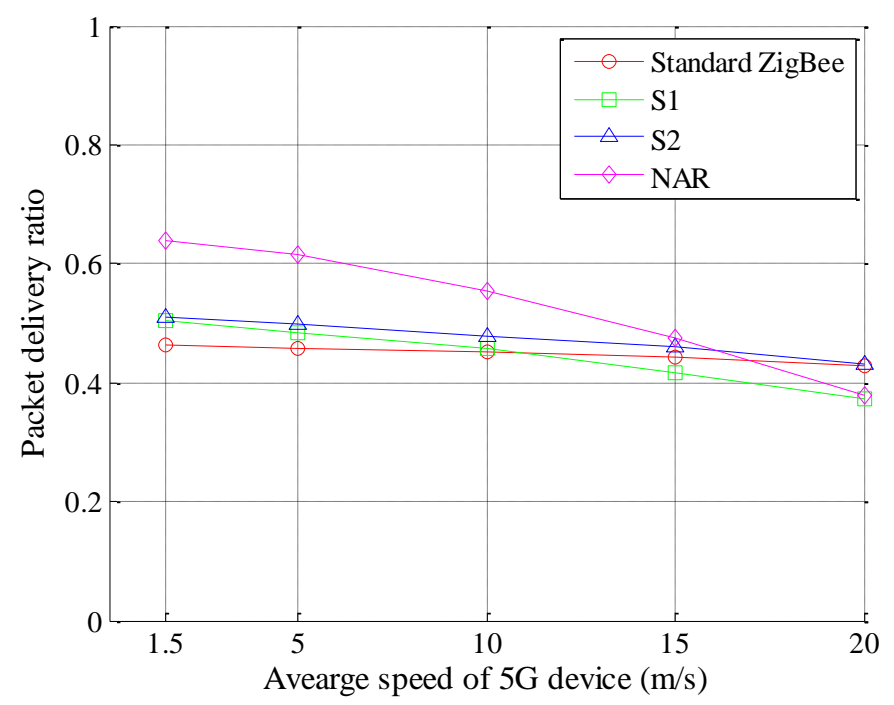

(a)

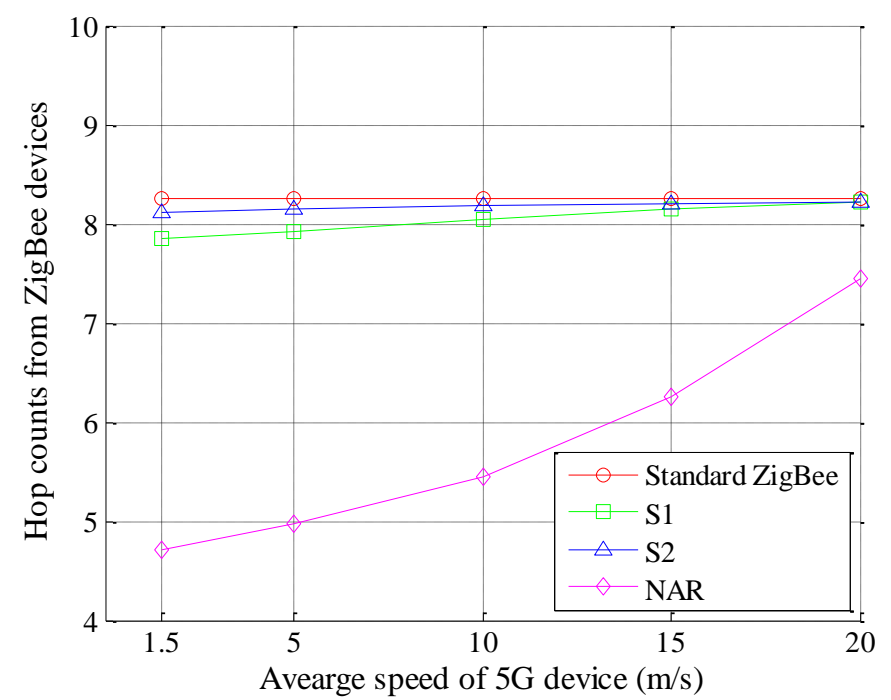

(b)

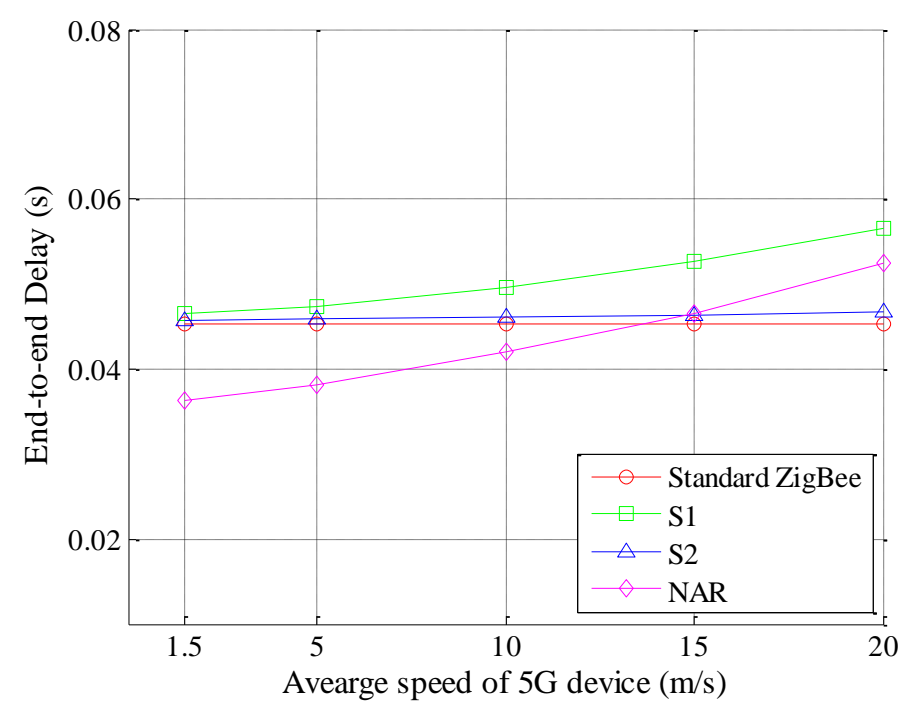


(c)

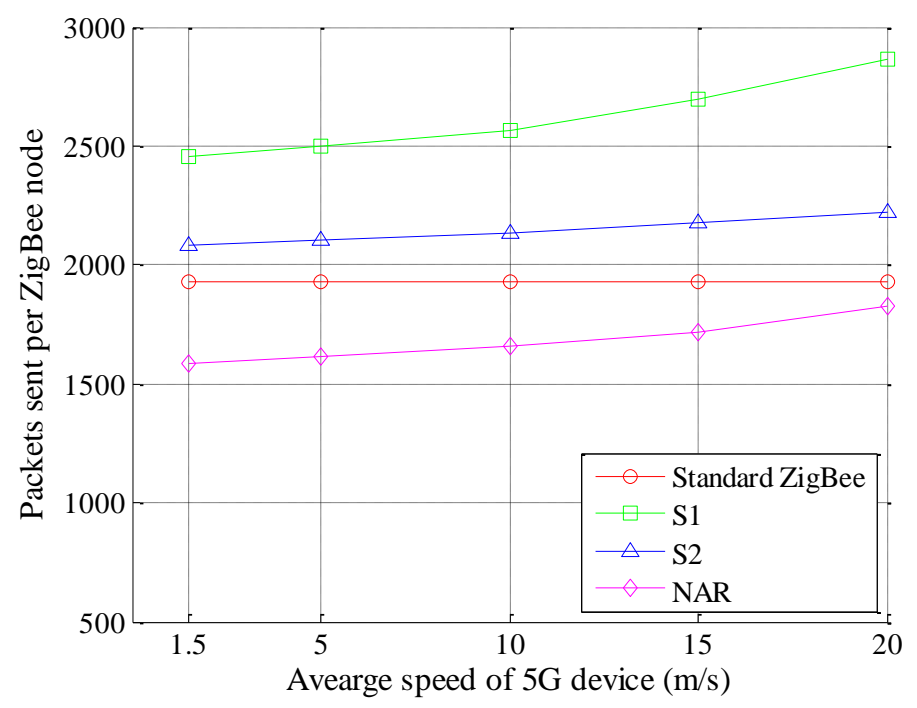

(d)

Fig.8. Performance evaluation for different scenarios with different $5 \mathrm{G}$ node mobility

(a) packet delivery ratio (b) hop counts (c) end-to-end delay (d) packets sent per ZigBee node

\section{Conclusion}

In this paper, we focus on the routing improvement on ZTR in 5G environment. The feasibility is based on the the smarter devices and the native support for the M2M communication, which are the features of $5 \mathrm{G}$ communication. We firstly develop two scenarios in which the topology updating mechanism is considered to make the $5 \mathrm{G}$ devices are capable of optimize the transmission paths after their joining. Based on the performance analysis, the device type of 5G nodes is determined the routers and the idea of gathering packets in their neighbourhoods by mobile terminals is developed. Then the nearest access routing algorithm is proposed. By introducing the physical depth and designing its maintenance mechanism, 5G devices are recognized and the optimized links via them are also discovered. Afterwards, the improved routing methods for different communication types are discussed and the NAR algorithm is generalized. The simulations show that NAR achieves better performances with higher packet delivery ratio, less hop counts from ZigBee devices and lower end-to-end delay, while its overheads are reduced as well, the number of packets per ZigBee node sends is reduced and it has zero routing overheads for ZigBee devices. Besides, the effect of $5 \mathrm{G}$ nodes mobility on the improvement is also investigated. The results indicate that NAR is more sensitive to the mobility due the packets gathering by $5 \mathrm{G}$ devices. Although the performances deteriorate faster, even for the worst case in our simulations, NAR still benefits hop counts and the packets sent per ZigBee node, while the other metrics are no worse than the level of other scenarios. The improvements both on the network performances and overheads imply that the $5 \mathrm{G}$ devices effectively share the communication in ZigBee networks in NAR.

For our further works, since the mobility of $5 \mathrm{G}$ device affects the performance much and the devices may have different levels of mobility in real world, we are to design an adaptive routing methods in which the low mobility $5 \mathrm{G}$ nodes gather and retransmit the packets and the devices 
with high speed only collect the information of ZigBee nodes deployment to optimize the path selection.

Acknowledgements: This work is funded by the National Science Foundation of China, NSFC: 61401301 and NSFC: 61401309

\section{References:}

[1] ZigBee Standard Organization, ZigBee Specification Document 053474r17 (ZigBee Alliance, San Ramon, 2007)

[2] IF Akyildiz,W Su,Y Sankarasubramaniam,E Cayirci. "Wireless sensor networks: A survey", Computer Networks, 2002, 38(4):393 - 422.

[3] Perkins, Charles E., and E. M. Royer. "Ad-hoc On-Demand Distance Vector Routing." The Workshop on Mobile Computing Systems \& Applications 1999:90--100.

[4] F Cuomo, S Della Luna, U Monaco, F Melodia. "Routing in ZigBee: Benefits from Exploiting the IEEE 802.15.4 Association Tree”, IEEE International Conference on Communications, 2007:3271-3276.

[5] Tung, Hoi Yan, et al. "The Generic Design of a High-Traffic Advanced Metering Infrastructure Using ZigBee." IEEE Transactions on Industrial Informatics 10.1(2014):836-844.

[6] M Tekkalmaz,I Korpeoglu. "PSAR: power-source-aware routing in ZigBee networks", Wireless Networks, 2012, 18(6):635-651.

[7] Huang, Yu Kai, et al. "Distributed Throughput Optimization for ZigBee Cluster-Tree Networks." IEEE Transactions on Parallel \& Distributed Systems 23.3(2011):513-520.

[8] Setiawan, Made A., et al. "ZigBee-Based Communication System for Data Transfer Within Future Microgrids." IEEE Transactions on Smart Grid 6(2015):1-1.

[9] Tsang, Kim Fung, M. Gidlund, and J. Åkerberg. "Guest Editorial Industrial Wireless Networks: Applications, Challenges, and Future Directions." IEEE Transactions on Industrial Informatics 12.2(2016):755-757.

[10] F Boccardi,RW Heath,A Lozano,TL Marzetta. "Five disruptive technology directions for 5G", IEEE Communications Magazine, 2014, 52(2):74-80

[11] Palattella, Maria, et al. "Internet of Things in the 5G Era: Enablers, Architecture and Business Models." IEEE Journal on Selected Areas in Communications 34.3(2016):1-1.

[12] Ramírez, Diego F., and S. Céspedes. "Routing in Neighborhood Area Networks: A survey in the context of AMI communications." Journal of Network \& Computer Applications 55.10(2015):68-80.

[13] J G. Andrews, S Buzzi, W Choi, SV. Hanly, AC. K. Soong, JC Zhang. "What Will 5G Be?”, IEEE Journal on selected areas in communications, 32(6): June 2014, pp 1065-1082.

[14] Gohil, A., H. Modi, and S. K. Patel. "5G technology of mobile communication: A survey." International Conference on Intelligent Systems and Signal Processing 2013:288 - 292.

[15] Chen, Ling Jyh, T. Sun, and N. C. Liang. "An Evaluation Study of Mobility Support in ZigBee Networks." Journal of Signal Processing Systems 59.1(2010):111-122.

[16] WH Chung,PC Hsiu,YY Shih,AC Pang,YK Huang,etc. "Mobility-Robust Tree Construction in ZigBee Wireless Networks", IEEE International Conference on Communications, 2011, 41(4):1-6.

[17] Abbasi, Ameer Ahmed, and M. Younis. "A survey on clustering algorithms for wireless sensor networks." Computer Communications 30.14-15(2007):2826-2841. 
[18] Kim, Taehong, et al. "Neighbor Table Based Shortcut Tree Routing in ZigBee Wireless Networks." IEEE Transactions on Parallel \& Distributed Systems 25.3(2014):706-716.

[19] Wadhwa, L. K., R. S. Deshpande, and V. Priye. "Extended shortcut tree routing for ZigBee based wireless sensor network." Ad Hoc Networks 37.P2 (2015):295-300.

[20] Kim, Taehong, and D. Kim. "Opportunistic Shortcut Tree Routing in ZigBee Networks." IEEE Sensors Journal 16.12(2016):5107-5115.

[21] Huang, Yu Kai, et al. "Distributed Throughput Optimization for ZigBee Cluster-Tree Networks." IEEE Transactions on Parallel \& Distributed Systems 23.3(2011):513-520.

[22] M Kasraoui,A Cabani,J Mouzna. "Improvement of Zigbee Routing Protocol”, 2012 IEEE International Conference on Green Computing and Communications, Conference on Internet of Things, and Conference on Cyber, Physical and Social Computing, 788-793.

[23] Shih, Yuan Yao, et al. "A Mobility-Aware Node Deployment and Tree Construction Framework for ZigBee Wireless Networks." IEEE Transactions on Vehicular Technology 62.6(2013):2763-2779.

[24] De Melo, P. O. S. V., F. D. Cunha, and A. A. F. Loureiro. "A distributed protocol for cooperation among different wireless sensor networks." IEEE International Conference on Communications IEEE, 2013.

[25] Akkaya, Kemal, and M. Younis. A survey on routing protocols for wireless sensor networks. Sustainable Wireless Sensor Networks. 2010:572-581.

[26] Perillo, Mark, Z. Cheng, and W. Heinzelman. "On the problem of unbalanced load distribution in wireless sensor networks." Global Telecommunications Conference Workshops, 2004. GLOBECOM Workshops IEEE, 2004:74-79.

[27] R Some. "A Novel Routing Protocol using Heterogeneous Zigbee Modules for Mobile Sensor Network", 2015 International Conference on Advances in Computing, Communications and Informatics (ICACCI), 2096-2102.

[28] Oh, Hoon. "A tree-based approach for the Internet connectivity of mobile ad hoc networks." Journal of Communications \& Networks 11.3(2009):261-270.

[29] Hong, Sung Hwa, and B. K. Kim. "An efficient data gathering routing protocol in sensor networks using the integrated gateway node." IEEE Transactions on Consumer Electronics 56.2(2010):627-632.

[30] Kinoshita, K., et al. "Fair Routing for Overlapped Cooperative Heterogeneous Wireless Sensor Networks." IEEE Sensors Journal 16.10(2016):1-1.

[31] Y Shen,Q Pei,N Xi,J Ma,Q Xu. "HGRP Hybrid Grid Routing Protocol for Heterogeneous Hierarchical Wireless Networks", 2011 Third International Conference on Intelligent Networking and Collaborative Systems, 320-327.

[32] Guidoni, Daniel Ludovico, et al. "RouT: A Routing Protocol based on Topologies for Heterogeneous Wireless Sensor Networks." IEEE Latin America Transactions 12.4(2014):812-817.

[33] Oyman, E. I., and C. Ersoy. "Multiple sink network design problem in large scale wireless sensor networks." IEEE International Conference on Communications 2004:3663-3667 Vol.6.

[34] Fabbri, F., C. Buratti, and R. Verdone. "A Multi-Sink Multi-Hop Wireless Sensor Network Over a Square Region: Connectivity and Energy Consumption Issues." GLOBECOM Workshops 2009:1-6. 